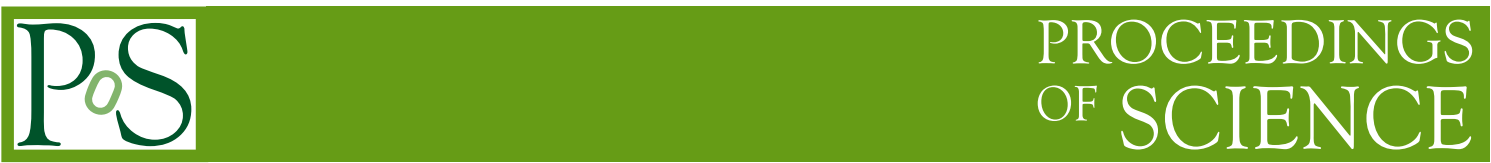

\title{
Quest for Dark Matter with Cosmic Gamma-ray Observations
}

\section{Hiroyasu Tajima*}

Solar-Terrestrial Environment Laboratory, Nagoya University

E-mail: tajima@nagoya-u.jp

\begin{abstract}
The dark matter is now widely accepted as a major ingredient of the Universe and considered to constitute 6 times as much as the ordinary matter. However, the nature of the dark matter remains one of the biggest mysteries in the Universe. Since there is no plausible theory for the dark matter at this moment, we have variety of dark matter candidates, posing significant experimental challenges for detection and measurement of their properties. Among the dark matter candidates, Weakly Interacting Massive Particles (WIMPs) are the leading candidate since the scale of expected mass and cross section well matches the new physics expected in the weak scale such as supersymmetry and extra dimension. Gamma-ray observations aim at detecting gamma rays produced by WIMP annihilations or decays, and would provide direct constraints on the annihilation cross section which can be compared with the value expected from the thermal relic hypothesis. Current gamma-ray experiments exclude the WIMP mass below $20 \mathrm{GeV} / c^{2}$ and future observations will be sensitive up to $>10 \mathrm{TeV} / c^{2}$ which covers a majority of the parameter space expected from new physics.
\end{abstract}

KMI International Symposium 2013 on "Quest for the Origin of Particles and the Universe", 11-13 December, 2013

Nagoya University, Japan

\footnotetext{
* Speaker.
} 


\section{Introduction}

Discovery of the Higgs particle by LHC experiments[1,2] completed the last missing piece of the Standard Model of particle physics in 2012. However, the Standard Model is known to have several issues such as hierarchy problem that requires large quantum corrections for Higgs mass, inconsistency with gravity and too many parameters that cannot be derived from the first principle, which would require new physics (= new particles) in the $\mathrm{TeV}$ energy region. Extensive searches for new particles in $\mathrm{TeV}$ region have been performed in many front and yielded no direct evidence so far. In the energy frontier, LHC experiments did not find any new particles up to $\sqrt{s}=7 \mathrm{TeV}$. In the intensity frontier, many experiments attempted to detect loop effects in rare decays suppressed or prohibited by the Standard Model or in deviation from the Standard Model by the precision measurement of parameters such as electric and/or magnetic dipole moments of muons and neutrons. Although the g-2 measurement[3] found a possible evidence of the physics beyond the Standard Model, it has not been confirmed by other experiments.

In the cosmic frontier, a combination of the measurements of anisotropy of cosmic microwave backgrounds, baryon acoustic oscillations and distance of Type Ia supernovae establishes the $\Lambda$ CDM (cold dark matter) model[4] as the Standard Model of cosmology where 'cold' refers to nonrelativistic speed of the dark matter. However, the nature of the main ingredients of the Universe, the dark energy $(=\Lambda)$ and the dark matter, remains one of the biggest mysteries in the Universe and may also hold a key to find new physics beyond the Standard Model of particle physics.

Besides the $\Lambda$-CDM model, the dark matter is now widely accepted as a major ingredient of the Universe through many pieces of evidence such as the orbital velocities of stars in galaxies, and the velocity dispersions of galaxies in clusters of galaxies, the temperature distribution of hot gas and the gravitational lensing of the background light in clusters of galaxies. The dark matter is considered to constitute $\sim 6$ times as much as the ordinary matter (stars and interstellar gas)[5] and dominate the gravitational effects by the matter.

Since there is no plausible theory for the dark sector, we have variety of candidates for them, posing significant experimental challenges for detection and measurement of their properties. Properties of the cold dark matter include non-relativistic speed at the epoch of radiation-matter equality, possibly non-baryonic, non-radiating and collisionless (i.e., only gravitational interactions). Leading cold dark matter candidates include Massive Compact Halo Objects (MACHOs), axions and WIMPs (Weakly Interacting Massive Particles). MACHOs can be black holes, neutron stars, brown dwarfs or unassociated planets. Since MACHOs are baryonic, they must be formed in the very early Universe to avoid conflicts with baryon acoustic oscillations, anisotropy of the cosmic microwave background and large-scale structure of galaxies. Gravitational microlensing surveys such as MACHO, EORS, MOA and OGLE placed severe constraints such that MACHOs cannot account for the large fraction of the dark matter[6,7]. The axion is introduced to avoid a large $C P$ violation in strong interactions. Although the axion is light, $\lesssim \mathrm{eV}$, it can be produced in a non-thermal process, and thus non-relativistic. WIMPs can be neutralino (or the lightest neutral supersymmetric particle), majorana fermion, sterile neutrino or Kaluza-Klein excitations. In general, WIMPs are considered to be thermal relics from the early Universe where they are produced in the hot plasma at the temperature greater than the WIMP mass. WIMPs continue annihilations after the temperature dropped below their mass due to the cooling of the Universe until the WIMP's number density 
becomes low enough compared with the annihilation cross section. With this assumption and the WIMP density at the freeze-out, the velocity-averaged annihilation cross section of WIMP can be calculated as

$$
\left\langle\sigma_{\mathrm{A}} v\right\rangle=2.8 \times 10^{-26} \mathrm{~cm}^{3} / s
$$

The cross section is coincidentally in the vicinity of the weak interaction cross section for WIMPs of mass at the electroweak energy scale (around a few hundred $\mathrm{GeV}$ ). Considering the vast parameter space ( $>70$ order of magnitudes) for the possible dark matter properties, this coincidence is often referred as "WIMP miracle" making WIMPs the most plausible candidates for the cold dark matter. In another word, if we have new particles around the $\mathrm{TeV}$ region, and one of them is neutral and stable, it will inevitably become a dark matter. This means that the detection of the WIMP dark matter represents a major breakthrough in the quest for new physics beyond the Standard Model of particle physics as well as providing a major missing piece of the Standard Model of cosmology.

Searches for the WIMP dark matter involve multi-pronged approach since the parameter space of the WIMP properties such as mass and cross section (annihilation, elastic scattering and pair creation) is very large ranging several order of magnitudes. Accelerator experiments can produce WIMP particles down to very small pair creation cross section. Once WIMP particles are detected by accelerator experiments, their mass and cross section can be measured very precisely. A major drawback of the accelerator experiments is limited mass range (up to $\sim \mathrm{TeV} / c^{2}$ ) due to the capability of the extremely expensive accelerator, i.e. Large Hadron Collider. Direct search experiments intend to detect nuclear recoils caused by local WIMP particles in underground detectors in very low-background environments. They are sensitive for WIMPs in a wide mass range, in particular in the higher mass range above $\mathrm{TeV} / \mathrm{c}^{2}$. On the other hand, direct search experiments suffer wide variety of backgrounds at low recoil energies $(\sim 10 \mathrm{keV})$, which yielded conflicting detections and exclusions at the relatively low mass region around $\sim 10 \mathrm{GeV} / c^{2}$. Indirect search experiments aim at detecting particles produced by the WIMP annihilations or decays, such as gamma rays, positrons, anti-protons and anti-deuterons. Indirect search experiments provide direct constraints on the annihilation cross section which can be compared with the value expected from the thermal relic hypothesis. Gamma rays are the most promising messenger to search for the WIMP annihilations since it is not deflected by interstellar magnetic filed unlike charged particles and it can be detected with high efficiencies unlike neutrinos. Since gamma rays can be traced to the WIMP annihilation site, gamma-ray searches can also provide the WIMP distribution in the Universe (most likely nearby or in our Galaxy) once we detect the signature.

In this manuscript, searches for gamma-ray signatures from the WIMP annihilations by currently operating gamma-ray instruments are reviewed and prospects for future observations are discussed.

\section{Searches for gamma-ray signatures from WIMP annihilations}

Searches for gamma-ray signatures from the WIMP annihilations involve multi-pronged approaches due to uncertainties of the WIMP density profile and annihilation cross section, and varying gamma-ray backgrounds. 
- Galactic Center is expected to be the brightest in the gamma-ray emission from the WIMP annihilations, however, we also have bright gamma-ray sources there.

- Galactic Halo is also expected to be relatively bright in the diffuse gamma-ray emission from the WIMP annihilations with modest Galactic diffuse backgrounds (except Galactic plane).

- Galactic subhalos and satellite galaxies (dwarf spheroidal galaxies) of Milky Way such as Sagittarius, Segue1 and Draco have a large dark matter content, 10-100 times higher than luminous matter without active star formation or detected gas content, which provides very low background environment. Since the gamma-ray flux from this class of sources is small, it requires very sensitive gamma-ray instrument (and/or long observation time) to detect such gamma-ray signatures.

- WIMP gamma-ray signatures in extragalactic sources such as galaxy clusters and isotropic gamma-ray emission (due mostly to unresolved extragalactic sources) are very faint and suffers backgrounds from baryonic matter (mostly active galactic nuclei).

In the electroweak scale mass region $\left(\mathrm{GeV} / c^{2}-\mathrm{TeV} / c^{2}\right)$, Large Area Telescope onboard Fermi Gamma-ray Space Telescope (Fermi-LAT)[8] launched in 2008 and Imaging Atmospheric Čerenkov Telescopes (IACTs) such as H.E.S.S.[9] (since 2004), MAGIC[10] (two telescopes since 2009) and VERITAS[11, 12] (four telescopes since 2007) are the most sensitive experiments currently in operation.

\subsection{WIMP searches by Fermi-LAT}

Below $\sim 1 \mathrm{TeV} / c^{2}$, searches for gamma-ray signatures from dwarf spheroidal galaxies and the Milky Way Halo region by Fermi-LAT provides the most stringent constraints[13, 14] thanks Fermi-LAT's large field of view ( $\sim 20 \%$ of the sky), high duty cycle $(>90 \%)$ with a modest active area $\left(>1.9 \mathrm{~m}^{2}\right)$.

Dwarf spheroidal galaxies are one of the most promising candidates to detect gamma-ray signatures from the WIMP annihilations since they are among the most dark-matter dominated objects known. In the recent LAT analysis, no gamma-ray signal is found in the direction of 25 dwarf spheroidal galaxies with few backgrounds in four years of observations. Relating the gamma-ray flux to the annihilation cross section depends on the assumption on the WIMP density and velocity distributions and possible final states from the WIMP annihilations. The $J$-factor, the line-of-sight integral of the dark matter density squared in a solid angle, can be calculated for 18 of dwarf galaxies whose dynamical mass can be constrained by stellar kinematic data sets using the prescription of Martinez[15], which is fairly insensitive to the dark matter density profiles, a Navarro-Frenk-White (NFW) profile[16] and a cored Burkert profile[17] assumed in this analysis. The NFW profile is used as a default and the effect of this choice is evaluated separately.

Assuming that the WIMP properties are common across the dwarf galaxies, gamma-ray data from selected dwarf galaxies are combined by forming a joint likelihood function from the product of the individual likelihood function for each dwarf galaxy. In this process, three dwarf galaxies are eliminated since their analysis regions overlap with other dwarf galaxies with larger $J$-factors, i.e. larger total WIMP mass, leaving 15 dwarf galaxies for the combined analysis. Since no significant 


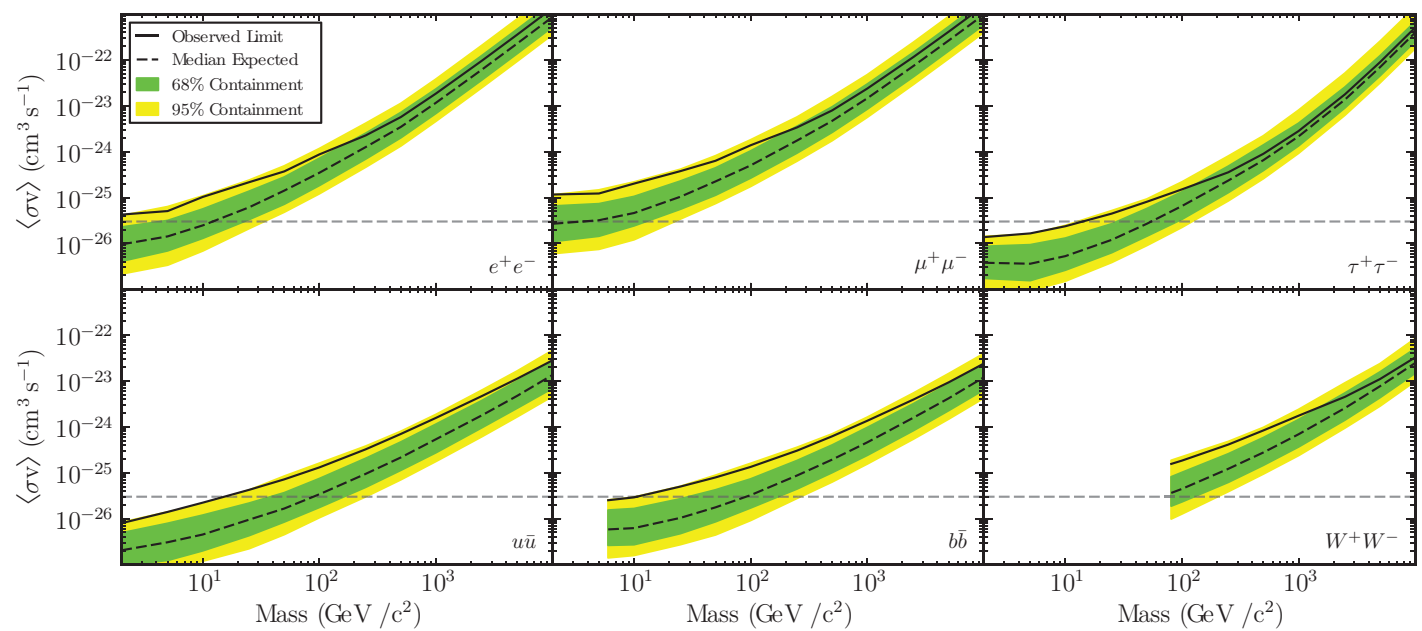

Figure 1: The solid line shows 95\% confidence level (C.L.) upper limits on the WIMP annihilation cross section for the selected 15 dwarf spheroidal galaxies and for annihilation into the $e^{+} e^{-}, \mu^{+} \mu^{-}, \tau^{+} \tau^{-}, u \bar{u}$, $b \bar{b}$ and $W^{+} W^{-}$channels assuming an NFW dark matter profile. The dashed line and the associated green and yellow bands show the median expected sensitivity, and the $68 \%$ and $95 \%$ quantiles, respectively. These limits include uncertainties associated with WIMP density profiles. The WIMP annihilation cross section expected from the thermal relic hypothesis is also shown as a reference.

signal is found for any annihilation channels, $e^{+} e^{-}, \mu^{+} \mu^{-}, \tau^{+} \tau^{-}, u \bar{u}, b \bar{b}$ and $W^{+} W^{-}$, in the combined analysis, 95\% confidence level (C.L.) upper limits on the WIMP annihilation cross section are calculated by finding the gamma-ray flux where the log-likelihood is smaller than its maximum by a factor of $e / 2$. Fig. 1 shows the combined 95\% C.L. upper limits on the WIMP annihilation cross section for the $e^{+} e^{-}, \mu^{+} \mu^{-}, \tau^{+} \tau^{-}, u \bar{u}, b \bar{b}$ and $W^{+} W^{-}$channels. These upper limits include uncertainties associated with WIMP density profiles, which increases the limit by $\sim 15 \%$ for the combined limit. The WIMP annihilation cross section expected from the thermal relic hypothesis is also shown as a reference. This result excludes the WIMP mass up to $\sim 10 \mathrm{GeV} / c^{2}$ for the $b \bar{b}$ channel and $\sim 15 \mathrm{GeV} / c^{2}$ for the $\tau^{+} \tau^{-}$channel. IACTs are less competitive than Fermi-LAT for the analysis of dwarf spheroidal galaxies due to limited observation time with small field of view. Fermi-LAT's all-sky survey capability is a great advantage for combining the signals from multiple sources. Moreover, the Fermi-LAT analysis can include more dwarf spheroidal galaxies that will be discovered in the future.

The Fermi-LAT collaboration also searched for gamma-ray signatures from the WIMP annihilations in the diffuse gamma-ray emission from the Milky Way Halo region (within $15^{\circ}$ in Galactic latitude and within $80^{\circ}$ in longitude)[14]. In this analysis, Galactic center and disk region (within $5^{\circ}$ in Galactic latitude) is excluded to avoid the the bright sources and diffuse emissions, which also reduces uncertainties due to the modeling of the WIMP profile. In this region, the gamma-ray emissions are dominated by the foreground astrophysical diffuse emissions originated from cosmic rays interacting with interstellar gas, cosmic-ray electrons interacting with interstellar radiation field. The GALPROP[18] v54 is used to model the astrophysical diffuse emissions, which accounts for the propagation of cosmic rays, the production of secondary particles in cosmic-ray interactions and the reacceleration of cosmic rays. The WIMP density profile is modeled by an NFW profile or 

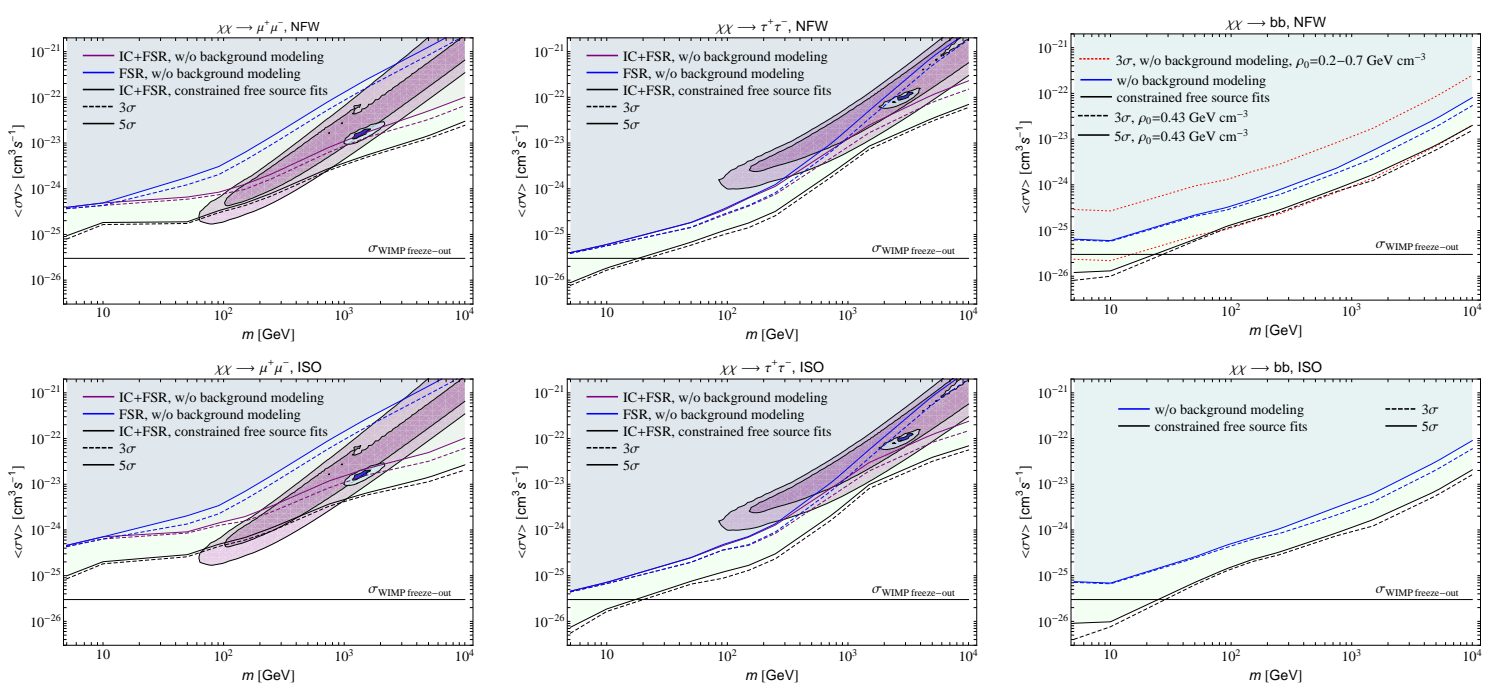

Figure 2: Dashed and solid lines show $3 \sigma$ and $5 \sigma$ C.L. upper limits, respectively, on the WIMP annihilation cross sections for $\mu^{+} \mu^{-}$(left panels), $\tau^{+} \tau^{-}$(middle panels) and $b \bar{b}$ (right panels) channels with different WIMP density profiles, NFW (top panels) and isothermal (bottom panels) profiles.

(Left+middle panels) Purple and blue contours indicate the WIMP mass and annihilation cross section consistent with PAMELA positron[21] and Fermi-LAT electron+position[22] spectra.

(Right panels) Blue lines show limits without diffuse emission model for $b \bar{b}$ channels.

(Top right panel) Red lines show $3 \sigma$ C.L. upper limits for the local WIMP densities, $\rho_{0}=0.2$ and $0.7 \mathrm{GeV} / \mathrm{cm}^{3}$ without the background modeling.

a cored (isothermal-sphere) profile[19, 20], which roughly brackets the uncertainties of the WIMP density profile.

The fit procedure is performed in two steps since extremely intensive computation is required to fit spatial and spectral distributions of gamma rays globally while all WIMP and astrophysical model parameters are varied freely. In the first step, some parameters (halo heights and diffusion coefficients) are constrained to 6 sets of models based on the local cosmic-ray measurements. In the second step, the gamma-ray data is fit using remaining model parameters. Upper limits for the WIMP contribution is calculated by finding the parameter set where the likelihood function is decreased by a factor of $\exp \left(3^{2} / 2\right)$ from the maximum likelihood for $3 \sigma(99.7 \%$ C.L.) , and $\exp \left(5^{2} / 2\right)$ for $5 \sigma(99.99994 \%$ C.L.). Fig. 2 shows the $3 \sigma$ and $5 \sigma$ upper limits on the WIMP annihilation cross section for the $\mu^{+} \mu^{-}, \tau^{+} \tau^{-}$and $b \bar{b}$ channels with different WIMP density profiles, NFW and isothermal profiles assuming the local WIMP density $\rho_{0}=0.43 \mathrm{GeV} / \mathrm{cm}^{3}[23]$. The uncertainty of $\rho_{0}=0.2-0.7 \mathrm{GeV} / \mathrm{cm}^{3}[23,24]$ is the dominant uncertainty for deriving the WIMP annihilation cross section. This result excludes the WIMP mass up to $\sim 20 \mathrm{GeV} / c^{2}$ for the $b \bar{b}$ and $\tau^{+} \tau^{-}$channels.

\subsection{WIMP searches by IACT}

Above $\sim 1 \mathrm{TeV}$, observations of Galactic Halo near Galactic Center (Galactic Center Halo) by the H.E.S.S. experiment provide the most stringent constraints[25] thanks to its large collection area of $\sim 2 \mathrm{~km}^{2}$. In this analysis, two regions are selected, one closer to the center with higher WIMP density and the other further from the center with lower WIMP density as shown in Fig. 3 
(a)

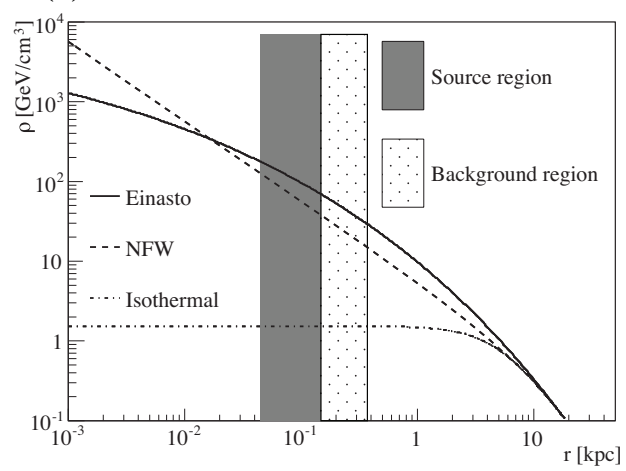

(b)

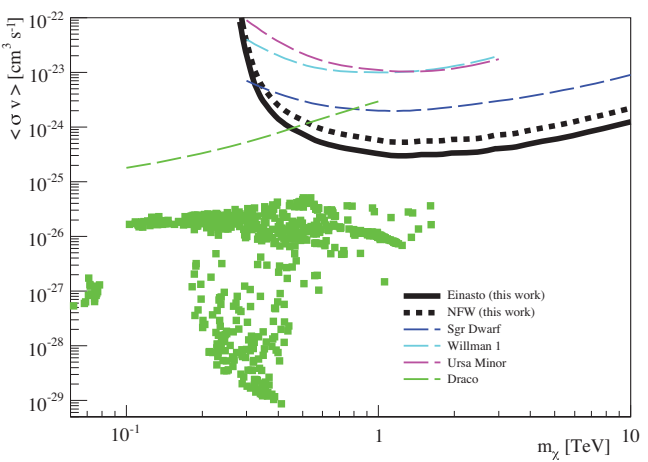

Figure 3: (a) Galactic WIMP halo profiles and two observation regions used in this analysis. The NFW and Einasto profiles are represented by dashed and solid curves. (b) 95\% C.L. upper limits on the WIMP annihilation cross section as a function of the WIMP mass for the $q \bar{q}$ final state. The limits for the Einasto and NFW density profiles are shown as black dashed and solid curves, respectively. Colored curves show limits for Sagittarius[26] (blue), Willman 1 (cyan), Ursa Minor[27] (magenta) and Draco[28] (green) dwarf spheroidal galaxies. Green points indicate the parameter space allowed by DarkSUSY models[29].

(a). Those two regions are chosen to minimize the dependence on the WIMP density profile (two possible WIMP density profiles are shown in the figure). All profiles are normalized to the local WIMP density, $0.39 \mathrm{GeV} / \mathrm{cm}^{3}$, at a distance of $8.5 \mathrm{kpc}$ from the Galactic Center. The difference of "gamma-ray" fluxes in two regions can be attributed to the WIMP annihilations since backgrounds are dominated by cosmic-ray electrons and isotropic. The spectra measured in the two regions agree well within the errors, resulting in a null measurement from which upper limits on the WIMP annihilation cross section can be determined. Fig. 3 (b) shows 95\% C.L. upper limits on the WIMP annihilation cross section as a function of the WIMP mass for the $q \bar{q}$ final state (almost equivalent to the $b \bar{b}$ channel in the Fermi-LAT analysis). The best limit is achieved at around $1 \mathrm{TeV}$ although it is still an order of magnitude higher than the cross section expected from the thermal relic hypothesis.

\section{Future Prospects}

In continued searches for gamma-ray signatures from the WIMP annihilations in GeV gammaray observations, the Fermi satellite will be operating for initially planned 5 years plus at least 2 years of extension, and possibly longer term. In addition, more dwarf spheroidal galaxies are being discovered due to improved detection techniques, increasing the analysis targets further. Moreover, understanding of Galactic diffuse emissions are improving steadily for the Galactic Halo analysis. In TeV gamma-ray observations, the next generation of IACT, Čerenkov Telescope Array (CTA)[30, 31] is being developed to realize 10 times better sensitivities than the currently operating IACTs. Fig. 4 shows the sensitivities on the WIMP annihilation cross section for the $b \bar{b}$ channel expected from 10-year observations of 30 dwarf spheroidal galaxies by Fermi-LAT and a 500-hour observation of Galactic Center Halo by CTA[32]. Future gamma-ray observations should be able to detect signatures from the WIMP annihilations up to $>10 \mathrm{TeV} / \mathrm{c}^{2}$. These sensitivities are very complimentary with accelerator experiments and direct WIMP search experiments as shown 
in Fig. 5 where the sensitivities of three different approaches are compared with effective operator approach[33]. While all approaches are complimentary, the gamma-ray searches provide unique windows in searching for WIMPs above a few hundreds $\mathrm{GeV} / \mathrm{c}^{2}$.

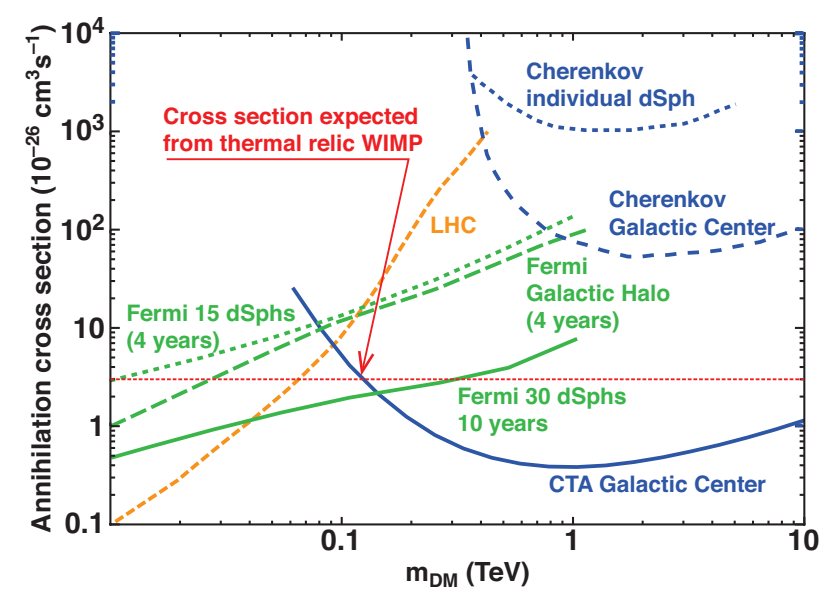

Figure 4: Sensitivities on the WIMP annihilation cross section as a function of the WIMP mass for the $b \bar{b}$ channel expected from 10-year observations of 30 dwarf spheroidal galaxies by Fermi-LAT (green) and CTA observations (500 hours) of Galactic Center Halo (blue). Current limits are shown in dotted and dashed lines.
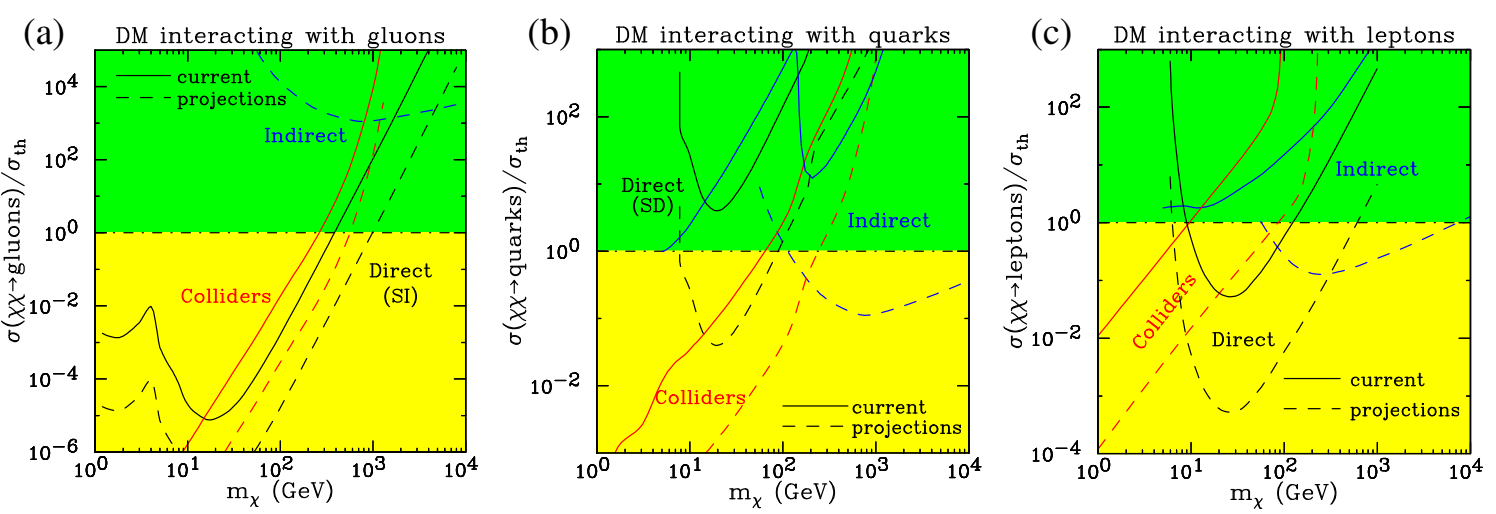

Figure 5: (a) Comparison of the sensitivities on the WIMP annihilation cross section among accelerator (collider) searches (red lines), spin-dependent direct searches (black lines) and indirect (gamma-ray observations) searches (blue lines) for the case the gluon coupling is dominant between WIMPs and standard model particles. (b) Comparison of the sensitivities for the case the quark coupling is dominant. In this case, direct searches are sensitive to only spin-independent scatterings. (c) Comparison of the sensitivities for the case the lepton coupling is dominant.

\section{References}

[1] G. Aad et al. Observation of a new particle in the search for the Standard Model Higgs boson with the ATLAS detector at the LHC. Phys. Lett. B716 (2012) 1. 
[2] S. Chatrchyan et al. Observation of a new boson at a mass of $125 \mathrm{GeV}$ with the CMS experiment at the LHC. Phys. Lett. B716 (2012) 30.

[3] G. Bennett et al. Final Report of the Muon E821 Anomalous Magnetic Moment Measurement at BNL. Phys. Rev. D73 (2006) 072003.

[4] J. P. Ostriker and P. J. Steinhardt. Cosmic Concordance. ArXiv Astrophysics e-prints astro-ph/9505066 (1995).

[5] E. Komatsu et al. Seven-year Wilkinson Microwave Anisotropy Probe (WMAP) Observations: Cosmological Interpretation. Astrophys. J. Suppl. 192 (2011) 18.

[6] C. Alcock et al. The MACHO Project: Microlensing Results from 5.7 Years of Large Magellanic Cloud Observations. Astrophys. J. 542 (2000) 281.

[7] C. Afonso et al. Limits on Galactic dark matter with 5 years of EROS SMC data. Astron. Astrophys. 400 (2003) 951.

[8] W. B. Atwood et al. The Large Area Telescope on the Fermi Gamma-Ray Space Telescope Mission. Astrophys. J. 697 (2009) 1071.

[9] H. E. S. S. collaboration. H.E.S.S. contributions to the 28th International Cosmic Ray Conference. ArXiv Astrophysics e-prints astro-ph/0307452 (2003).

[10] J. Albert et al. The MAGIC Project: Contributions to ICRC 2007. ArXiv e-prints 0709.3763 (2007).

[11] J. Holder et al. The first VERITAS telescope. Astropart. Phys. 25 (2006) 391.

[12] J. Holder et al. Status of the VERITAS Observatory. In F. A. Aharonian, W. Hofmann and F. Rieger (editors), American Institute of Physics Conference Series, volume 1085 of American Institute of Physics Conference Series. 657-660.

[13] M. Ackermann, A. Albert, B. Anderson, L. Baldini, J. Ballet, G. Barbiellini et al. Dark matter constraints from observations of 25 Milky Way satellite galaxies with the Fermi Large Area Telescope. Phys. Rev. D89 (2014) 042001.

[14] M. Ackermann, M. Ajello, W. B. Atwood, L. Baldini, G. Barbiellini, D. Bastieri et al. Constraints on the Galactic Halo Dark Matter from Fermi-LAT Diffuse Measurements. Astrophys. J. 761 (2012) 91.

[15] G. D. Martinez. A Robust Determination of Milky Way Satellite Properties using Hierarchical Mass Modeling. ArXiv e-prints 1309.2641 (2013).

[16] J. F. Navarro, C. S. Frenk and S. D. M. White. A Universal Density Profile from Hierarchical Clustering. Astrophys. J. 490 (1997) 493.

[17] A. Burkert. The Structure of Dark Matter Halos in Dwarf Galaxies. Astrophys. J. 447 (1995) L25.

[18] A. W. Strong, I. V. Moskalenko and O. Reimer. Diffuse Continuum Gamma Rays from the Galaxy. Astrophys. J. 537 (2000) 763.

[19] K. G. Begeman, A. H. Broeils and R. H. Sanders. Extended rotation curves of spiral galaxies - Dark haloes and modified dynamics. Mon. Not. R. Astron. Soc. 249 (1991) 523.

[20] J. N. Bahcall and R. M. Soneira. The universe at faint magnitudes. I-Models for the galaxy and the predicted star counts. Astrophys. J. Suppl. 44 (1980) 73.

[21] O. Adriani, G. C. Barbarino, G. A. Bazilevskaya, R. Bellotti, M. Boezio, E. A. Bogomolov et al. An anomalous positron abundance in cosmic rays with energies 1.5-100GeV. Nature 458 (2009) 607. 
[22] A. A. Abdo, M. Ackermann, M. Ajello, W. B. Atwood, M. Axelsson, L. Baldini et al. Measurement of the Cosmic Ray $e^{+}+e^{-}$Spectrum from $20 \mathrm{GeV}$ to $1 T$ TeV with the Fermi Large Area Telescope. Phys. Rev. Lett. 102 (2009) 181101.

[23] P. Salucci, F. Nesti, G. Gentile and C. Frigerio Martins. The dark matter density at the Sun's location. Astron. Astrophys. 523 (2010) A83.

[24] M. Cirelli, G. Corcella, A. Hektor, G. Hütsi, M. Kadastik, P. Panci et al. PPPC 4 DM ID: a poor particle physicist cookbook for dark matter indirect detection. J. of Cosmo. and Astropart. Phys. 3 (2011) 051.

[25] A. Abramowski et al. Search for a Dark Matter Annihilation Signal from the Galactic Center Halo with H.E.S.S. Phys. Rev. Lett. 106 (2011) 161301.

[26] F. Aharonian et al. Observations of the Sagittarius dwarf galaxy by the HESS experiment and search for a dark matter signal. Astropart. Phys. 29 (2008) 55.

[27] V. A. Acciari et al. VERITAS Search for VHE Gamma-ray Emission from Dwarf Spheroidal Galaxies. Astrophys. J. 720 (2010) 1174.

[28] A. A. Abdo et al. Observations of Milky Way Dwarf Spheroidal Galaxies with the Fermi-Large Area Telescope Detector and Constraints on Dark Matter Models. Astrophys. J. 712 (2010) 147.

[29] P. Gondolo, J. Edsjö, P. Ullio, L. Bergström, M. Schelke and E. A. Baltz. DarkSUSY: computing supersymmetric dark matter properties numerically. J. of Cosmo. and Astropart. Phys. 7 (2004) 8.

[30] M. Actis, G. Agnetta, F. Aharonian, A. Akhperjanian, J. Aleksić, E. Aliu et al. Design concepts for the Cherenkov Telescope Array CTA: an advanced facility for ground-based high-energy gamma-ray astronomy. Exp. Astron. 32 (2011) 193.

[31] B. S. Acharya, M. Actis, T. Aghajani, G. Agnetta, J. Aguilar, F. Aharonian et al. Introducing the CTA concept. Astropart. Phys. 43 (2013) 3.

[32] M. Wood, J. Buckley, S. Digel, S. Funk, D. Nieto and M. A. Sanchez-Conde. Prospects for Indirect Detection of Dark Matter with CTA. ArXiv e-prints 1305.0302 (2013).

[33] D. Bauer, J. Buckley, M. Cahill-Rowley, R. Cotta, A. Drlica-Wagner, J. Feng et al. Dark Matter in the Coming Decade: Complementary Paths to Discovery and Beyond. ArXiv e-prints 1305.1605 (2013). 